\title{
Infizierte Plattenosteosynthese
}

Ulf-Joachim Gerlach

\section{Einleitung}

Die Infektion nach plattenosteosynthetischer Versorgung einer Fraktur stellt eine schwerwiegende postoperative Komplikation dar. Die konsequente adäquate Therapie ist entscheidend für den Therapieerfolg.

Die Ursachen für die Entwicklung einer Infektion nach Plattenosteosynthese sind vielfältig und können unfallbedingt, iatrogen sein oder durch patienteneigene systemische Faktoren begünstigt werden. Weitere Faktoren sind die Keimbesiedelung und die Biofilmbildung.

\section{Pathophysiologie}

Die Häufigkeit einer postoperativen Infektion nach Elektiveingriffen wird je nach Literatur mit 1-2\% angegeben. Bei offenen Frakturen beträgt sie 1-5\%. Bei offenen Frakturen tritt eine Infektion je nach Schweregrad in 3-50\% (bei drittgradig offenen Frakturen) [1,10] auf. Entscheidend für die Infektentstehung bei offenen Frakturen ist die durch den Unfall bedingte lokale Keimkontamination und eine Minderung der lokalen und systemischen Immunabwehr durch das Trauma, der lokale Knochen- und Weichteilschaden und die dadurch gestörte Durchblutung von Knochen und Weichteilen [7]. Wenn die Keimeinschleppung ausreichend massiv und die Infektabwehr ausreichend gestört ist, kommt es zum Zusammenbruch der Infektabwehr und zur rapiden Keimvermehrung.

Die operative Versorgung von Frakturen stellt ein zusätzliches Infektionsrisiko dar. Die Manipulation an den durch den Unfall bereits vorgeschädigten Weichteilen, Knochen und Periost ist für den Organismus ein weiteres lokales und systemisches Trauma. Auch der falsch gewählte OPZeitpunkt, wie die Durchführung einer OP in der Schwellphase nach mehr als 6 Stunden nach dem Unfall, erhöht das Infektrisiko, ebenso die falsche Implantatwahl und die OP-Dauer (über 2 Stunden). Weitere, die Entstehung einer Entzündung nach Osteosynthese begünstigende Faktoren sind das Belassen von Wundhöhlen gefüllt mit Serom/Hämatom als idealer Nährboden für eine Keimvermehrung, das nicht konsequente Entfernen von Fremdkörpern wie Schmutzpartikeln, von bradytrophem Gewebe und aus dem Gewebeverbund gelöster Knochenparti- kel. Ein nicht durchgeführtes Ausschneiden kontusionierter Wundränder erhöht ebenfalls das Risiko für eine Infektentstehung.

Systemische patienteneigene Faktoren begünstigen die Entwicklung eines postoperativen Infekts. Von Relevanz sind Begleiterkrankungen wie Adipositas, Nikotin-und Alkoholabusus, Diabetes mellitus, Durchblutungsstörungen, Hauterkrankungen mit entsprechenden Effloreszenzen im Zugangsgebiet wie Psoriasis, Immunschwäche, einlaufende immunsuppressive Therapie und Erkrankungen aus dem rheumatoiden Formenkreis sowie konsumierende Erkrankung, Begleitinfektion und Strahlenschäden. Auch das Lebensalter über 65 Jahre erhöht das Risiko eines postoperativen Infekts. Je mehr Komorbiditäten vorliegen, desto höher ist die Wahrscheinlichkeit einer Infektentstehung [2].

Implantatfreier Knochen benötigt 100000000 Keime, Knochen mit Implantat 100000 Keime zur Infektion. Staphylococcus aureus verursacht nahezu die Hälfte der postoperativen Infektionen, aber auch weitere Staphylokokkenentitäten, Streptokokken sowie Pseudomonaden und Enterobacteriaceae können als Erreger identifiziert werden. Nach Implantation des Osteosynthesematerials versuchen die Bakterien, sich über Adhäsine an das Implantat zu binden. Sie bilden dort durch einen bis zu $40 \mu \mathrm{m}$ dicken Biofilm geschützte Kolonien. Der Biofilm bietet Schutz vor Antikörpern und Phagozytose. Zunächst erfolgt die Besiedelung durch die planktonische Form, d. h. hohe Stoffwechselrate und eine rasche Vermehrung. Die Abwehrreaktion des Körpers wird ausgelöst, typische klinische Infektsymptome können auftreten. In dieser Phase sind die Keime gegenüber Antibiotika sensibel.

Nach Entwicklung des Biofilms gehen die Bakterien in die sessile Phase über. In der sessilen Phase verlangsamen sich die biologischen Reaktionen, die Reproduktionsrate ist deutlich reduziert. Durch den Schutz des gebildeten Biofilms sind die Keime teilweise um den Faktor 1000 unempfindlicher gegenüber Antibiotika. Unter dem Schutz des Biofilms können die Bakterien Informationen, wie z.B. Resistenzen, austauschen und weitergeben $[3,6,8]$. In dieser Phase entziehen sich die Keime der körpereigenen Abwehr. Die durch den Biofilm gebildete „City of Microbes“ bietet den Bakterien einen Überlebensvorteil. Die Bakterien treten in synergistische Wechselbeziehun- 
gen. Es resultiert die bessere Versorgung mit verwertbaren Substraten, der Schutz vor chemischen und mechanischen Einflüssen, eine erhöhte Resistenz und der Austausch genetischer Informationen.

Chronische Infektionen (implantatassoziierte Infektionen) sind durch bakterielle Biofilme verursacht.

Unterschieden wird die frühe Infektion nach Plattenosteosynthese innerhalb der ersten 4 Wochen nach operativer Versorgung und die späte Infektion nach 4 Wochen.

\section{Diagnostik}

Zwischen der frühen und der späten Infektion nach Plattenosteosynthese ist hinsichtlich der Diagnostik unterschiedlich vorzugehen.

Die Diagnose der Frühinfektion ist eine klinische Diagnose. Die klassischen Entzündungszeichen wie Rubor, Calor, Dolor, Tumor und Functio laesa können bei einem Frühinfekt vorliegen. Nicht immer jedoch ist die Unterscheidung von einem lokalen Weichteilschaden nach vorausgegangenem Trauma möglich. Typischerweise berichtet der Patient postoperativ über prolongierte und stärker ausgeprägte Schmerzen. Laborchemisch ist der CRPWert erhöht, es fehlt der typische Abfall des CRP-Werts nach dem 5. postoperativen Tag. Bildgebende Verfahren liefern keine zusätzlichen Informationen der frühen Infektion nach Plattenosteosynthese. Ein Nativröntgenbild sollte trotzdem durchgeführt werden, um ggf. eine Implantatdislokation oder einen Plattenausriss ausschließen zu können, der eine ähnliche Schmerzsymptomatik und Schwellung hervorrufen könnte. Eine MRT-Untersuchung ist nicht indiziert. Die im MRT nach Kontrastmittelgabe zu erkennenden Veränderungen können sowohl unfallbedingt, OP-bedingt als auch durch den Infekt verursacht sein. Gleiches gilt für eine 3-Phasen-Skelettszintigrafie. Eine bakteriologische Untersuchung ist ebenfalls nicht zielführend. Für eine sinnvolle bakteriologische Untersuchung sollten Gewebeanteile entnommen werden, die für 14 Tage bebrütet werden müssten. Die operative Behandlung einer Frühinfektion nach Plattenosteosynthese stellt eine dringliche Indikation dar, sodass ein Abwarten der Bebrütung der bakteriologischen Abstriche sich verbietet.

Bei der chronischen Infektion nach Plattenosteosynthese steht die sorgfältige Anamnese (Primärbefund nach Trauma, Verlauf nach primärer Osteosynthese, systemische Risikofaktoren) und der klinische Befund am Anfang der Diagnostik. Als Laborparameter notwendig und ausreichend ist die Bestimmung der Leukozytenzahl und des CRP-Werts. Weitere Laborparameter wie Procalcitonin, Elastase oder auch Interleukin weisen keine höhere Sensitivität oder Spezifität auf, sind aber deutlich teurer. Eine ggf. notwendige Zusatzuntersuchung wie eine angiologische oder fachneurologische Untersuchung ist veranlassen. Zur Basisdiagnostik gehört die Röntgennativaufnahme der betroffenen Extremität in 2 Ebenen. Mögliche radiologische Kriterien einer chronischen Knocheninfektion sind Osteolysen, Destruktion, Sklerosezonen und periostale Appositionen, ebenso Lockerungssäume um einliegendes Osteosynthesematerial [9]. Der radiologische Befund hängt dem klinischen Befund 2-3 Wochen hinterher. Die Computertomografie dient dem Nachweis von Sequestern, Gaseinschlüssen sowie komplexer knöcherner Veränderungen. Die Computertomografie ist der MRT-Untersuchung deutlich überlegen in der Aussage hinsichtlich der knöchernen Stabilität und fraglicher Pseudarthrosenbildung. Die MRT-Untersuchung kann bei einliegendem Osteosynthesematerial aufgrund der entstehenden Artefaktbildung zu einer Einschränkung der Beurteilung führen. Eine Abschätzung des Ausmaßes der Infektion sowie insbesondere auch der umgebenden Weichteile ist trotz der Einschränkungen der MRT-Untersuchung möglich. Die Skelettszintigrafie ist aufgrund ihrer eingeschränkten Spezifität zur Diagnose einer chronischen Infektion nach Plattenosteosynthese nicht geeignet. Die Hauptindikation dient als Screeningmethode bei vorliegender Osteomyelitis bei der Suche nach dem Fokus.

\section{Therapie}

Die operative Behandlung der frühen Infektion unterscheidet sich von der Behandlung der Spätinfektion.

Der Frühinfekt stellt einen Notfall dar, die operative Behandlung ist zeitnah einzuleiten. Ziel der Behandlung ist eine dauerhafte Infektberuhigung, die Vermeidung der Chronifizierung. Die Operation sollte von einem versierten Operateur durchgeführt werden, das Vorgehen ist abhängig von der lokalen Situation. Aufgrund der Biofilmbesiedelung ist das einliegende Osteosynthesematerial nicht zu erhalten, deswegen ist bei stabiler Osteosynthese ein einmaliger Versuch des Erhalts der internen Osteosynthese mit Wechsel des Osteosynthesematerials indiziert $[4,5]$. Die einliegende Plattenosteosynthese ist über die ganze Länge darzustellen. Wenn die Primärversorgung über einen minimalinvasiven Zugang erfolgte, muss bei der Infektsanierung ein invasives Vorgehen gewählt werden. Die zwischen den Inzisionen liegenden Hautbrücken müssen ebenfalls eröffnet werden. Es hat ein radikales Débridement zu erfolgen mit Entfernung aller nekrotischen, minderdurchbluteten und/oder infizierten Gewebeanteile. Die einliegende Osteosyntheseplatte ist zu entfernen. Das Plattenlager ist zu säubern, die Schraubenlöcher sind zu kürettieren. Anschließend sollte eine ausgiebige Spülung mit steriler Ringer-Laktat-Lösung oder eine Jet-Lavage erfolgen. Es sind ausreichend Gewebeabradate für die bakteriologische Untersuchung zu 

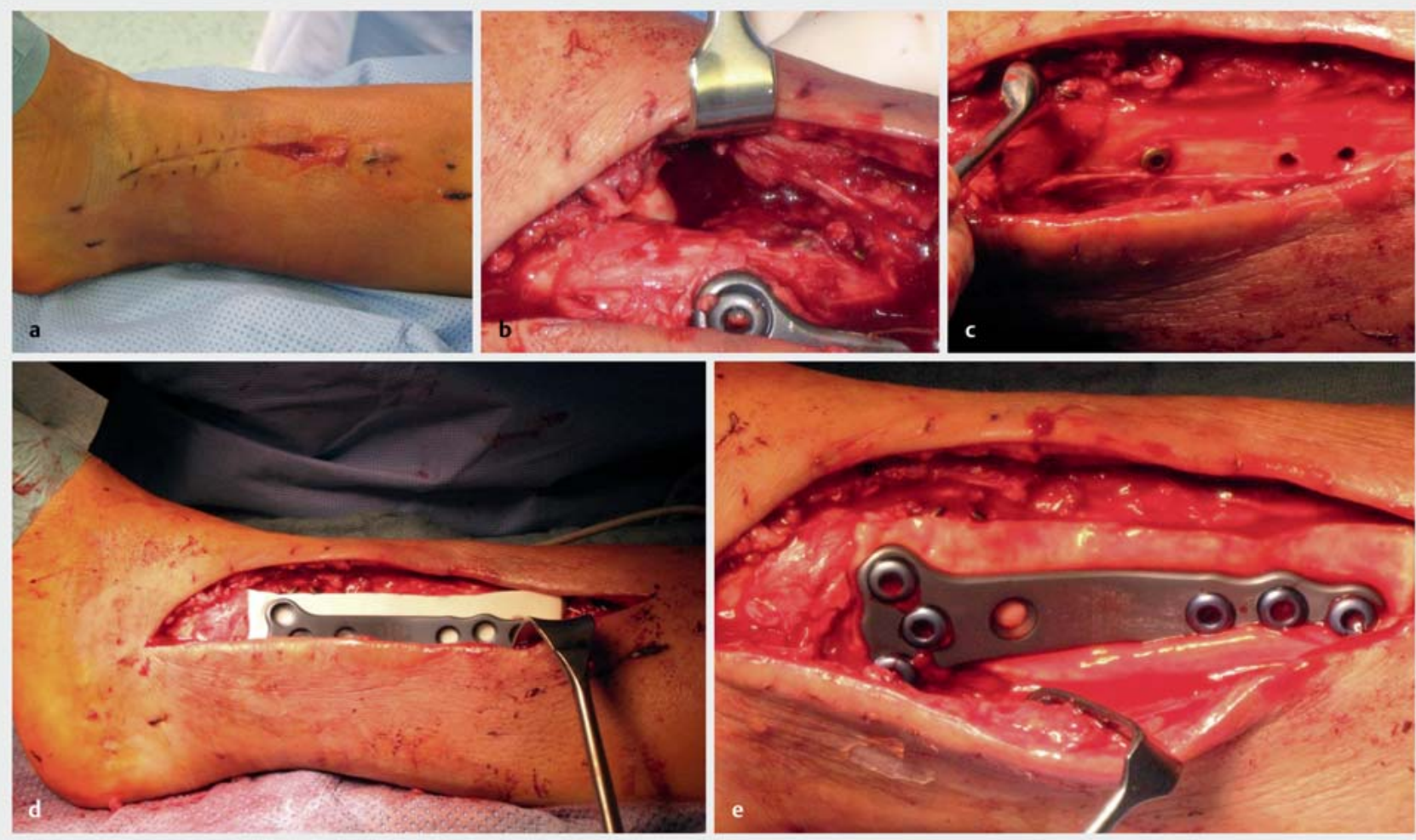

- Abb. 1 a Frühinfektion nach Osteosynthese einer Pilonfraktur mit Wunddehiszenz und Fistelung. b Arthrotomie OSG (Oberes Sprunggelenk) zum Ausschluss eines Empyems. c Débridement des Plattenlagers nach Entfernung der Osteosyntheseplatte, die interfragmentäre Zugschraube wurde noch entfernt. $\mathbf{d}$ Einlegen eines Gentamycin abgebenden Vlieses und einer neuen Osteosyntheseplatte. e Erfolgte Restabilisierung.

entnehmen. Es erfolgt dann die Restabilisierung mit einer neuen Osteosyntheseplatte. Auf das Plattenlager aufgelegt werden sollte ein Vlies, das lokal ein Antibiotikum (Gentamycin) abgibt ( $\bullet$ Abb. 1). Wenn die primäre Osteosynthese nicht stabil war, so ist im Falle des Infekts nach Entfernen der internen Osteosynthese eine Stabilisierung im Fixateur durchzuführen. Die Fraktur sollte dann im Fixateur ausbehandelt werden. Bei gelenknahen Frakturen hat immer eine Arthrotomie zu erfolgen. Das Gelenk muss inspiziert werden, um ein Empyem im Frühstadium auszuschließen. Sollte kein Gelenkinfekt vorliegen, so hat eine Spülung des Gelenks mit steriler RingerLaktat-Lösung zu erfolgen. Die Einlage eines Schwammes mit lokaler Antibiotikumabgabe hat zu erfolgen. Anders stellt sich die Situation dar bei Frühinfekt bei einliegendem Marknagel. Nach Entfernung des einliegenden Marknagels ist der Markraum aufzubohren mit nachfolgender Spülung des Markraums. Eine Restabilisierung mit einem erneuten Marknagel ist nicht empfehlenswert bei deutlichem Risiko der Reinfektion. Eine Stabilisierung im Fixateur externe und die Ausbehandlung mit einem Fixateur externe ist anzustreben. Eine geplante Second-Look-Operation führen wir nicht durch, der weitere Verlauf ist vom klinischen Befund abhängig. Die Etappenlavage, die wiederholte geplante operative Revision ist nicht indiziert und führt zu einer Verlängerung des Heilverlaufs für den Patienten. Wenn die Weichteile sich spannungsfrei oder spannungsarm verschließen lassen, ist ein primärer Verschluss anzustreben. Ein VAC-Verband sollte vermieden werden. Ein primär nicht zu verschließender Hautweichteildefekt sollte frühzeitig durch eine geeignete plastische Deckung definitiv verschlossen werden. Intraoperativ nach Abnahme der Abradate zur bakteriologischen Untersuchung sollte eine antibiotische Behandlung begonnen werden. Eine Doppelantibiose mit Rifampicin als ein Partner ist anzustreben, um eine Biofilmbildung zu vermeiden. Rifampicin sollte nicht als Monopräparat verwendet werden, um eine Resistenzentwicklung zu vermeiden. Nach Eingang der bakteriologischen Untersuchung ist dann ggf. die Antibiose anzupassen. Die antibiotische Behandlung sollte zunächst für 2 Wochen intravenös, dann für maximal 4 Wochen oral erfolgen.

Die Behandlung der chronischen Infektion bei Plattenosteosynthese ist deutlich aufwendiger. Die betroffenen Patienten wurden häufig mehrfach voroperiert, deswegen liegen nicht selten begleitende Haut-/Weichteildefekte vor sowie Inaktivitäts- oder Bewegungsmangelschäden. Die Behandlung hat nach einem Algorithmus zu erfolgen. Im 1. operativen Eingriff wird die notwendige 


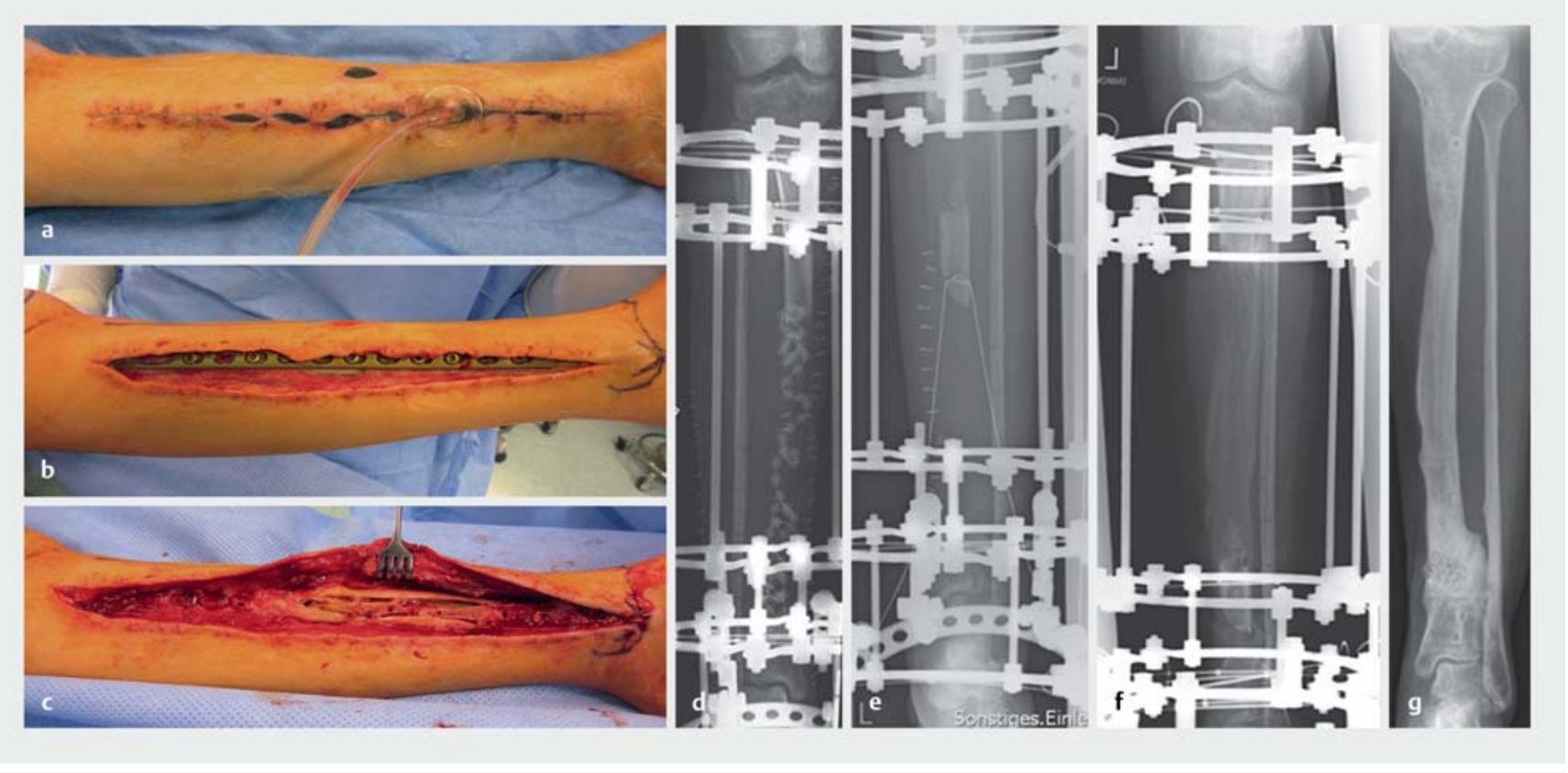

- Abb. 2 a Chronische Infektion nach Osteosynthese Tibia mit einliegendem Vakuumverband. b Klinischer Befund intraoperativ der infiziert einliegenden Osteosyntheseplatte nach Entfernung des Vakuumverbands. c Nach Entfernung der Ostesyntheseplatte - langstreckig sequestrierende Tibiaosteitis. d Röntgenbefund nach radikaler Sequestrektomie und Stabilisierung im Ringfixateur. e Laufender Segmenttransport von proximal nach distal. f Abgeschlossener Segmenttransport. g Ausheilungsergebnis.

radikale operative Behandlung zur Infektionsberuhigung durchgeführt. In einem 2. operativen Schritt ist ein möglicherweise vorliegender Weichteildefekt zu verschließen. Der 3. operative Schritt erfolgt bei Infektberuhigung zum Knochendefektaufbau. Parallel hat mit dem Tag der stationären Aufnahme ein umfassendes Rehaprogramm mit Physiotherapie, Sport- und Gehschultherapie, Hydro- und Ergotherapie zu erfolgen. Bei der Behandlung handelt es sich um eine multidisziplinäre Behandlung, die mehrzeitig durchzuführen ist. Im Rahmen des 1. operativen Schritts muss das einliegende Fremdmaterial entfernt werden. Eine radikale Sequestrektomie hat zu erfolgen [9]. Sämtliche entzündlichen oder avitalen Knochenanteile müssen entfernt werden. Zusätzlich muss ein Débridement der infizierten Weichteile durchgeführt werden. Lokale Antibiotikumträger (Vlies, antibiotikumabgebende Ketten, Knochenzement als Masqualet-Spacer) sind einzulegen. Bei resultierender Instabilität erfolgt die Stabilisierung durch einen externen Fixateur. Eine kurzzeitige systemische Antibiose ist erforderlich. Essenziell bei der Behandlung der Osteitis ist die radikale Sequestrektomie. Ohne Radikalität ist keine dauerhafte Infektberuhigung zu erzielen. Entscheidend ist die chirurgische Therapie, die lokale und systemische Antibiose sind lediglich unterstützend. Ein möglicherweise vorliegender Haut-/Weichteildefekt muss stabil und so früh wie möglich gedeckt werden. Hier finden alle gängigen Verfahren Anwendung, von der Dermatodistraktion, Verschiebelappenplastik bis hin zu einer freien Lappentransplantation mit mikrovaskulärem Anschluss. Bei sicherer Infektberuhigung - nach ca. 6 Wochen - erfolgt der nächste Schritt, die operative Behandlung des nach Sequestrektomie vorliegenden Knochendefekts. Ein zirkulärer Knochendefekt bis $3 \mathrm{~cm}$ wird durch autologe Spongiosaplastik nach Entnahme entweder vom hinteren Beckenkamm, vorderen Beckenkamm oder Tibiakopf durchgeführt. Ein zirkulärer Knochendefekt größer als $3 \mathrm{~cm}$ wird aufgebaut über einen Segmenttransport ( $\bullet \mathbf{A b b}$. 2). Eine alternative Methode stellt die Masqualet-Technik dar, die für kleinere Defekte eingesetzt wird. Ein freies vaskuläres Fibulatransplantat zum knöchernen Defektaufbau ist geeignet für obere Extremitäten oder untere Extremitäten bei Kindern. Nach radiologisch gesichertem Durchbau kann der Fixateur externe entfernt werden, die Versorgung mit einer teilentlastenden Orthese vor nachfolgender sukzessiver Belastungssteigerung ist sinnvoll.

\section{Schlussfolgerung}

Infektionen nach Plattenosteosynthese sind schwerwiegende Komplikationen, die zielgerichtet und konsequent behandelt werden müssen. Die inadäquate Behandlung führt zu einer deutlichen Verlängerung des Heilverlaufs mit erheblichen negativen Konsequenzen für den Patienten. Die operative Behandlung ist entscheidend und muss radikal erfolgen. Möglicherweise resultierende Knochen- und Weichteildefekte lassen sich in aller Regel 
wieder aufbauen. Die Behandlung sollte in einem Zenrum für septische Chirurgie erfolgen.

Interessenkonflikt

Nein

Über den Autor

\section{Ulf-Joachim Gerlach}

Dr., Abteilung für Septische Unfallchirurgie und Orthopädie (Chefarzt: Dr. U.-J. Gerlach), Berufsgenossenschaftliches Unfallkrankenhaus Hamburg (Ärztlicher Direktor: Prof. Dr. Ch. Jürgens)

\section{Korrespondenzadresse}

\section{Dr. Ulf-Joachim Gerlach}

Abteilung für Septische Unfallchirurgie und Orthopädie Berufsgenossenschaftliches Unfallkrankenhaus Hamburg Bergedorfer Straße 10

21033 Hamburg

Tel.: 0 40/7306-2413, Fax: 040/7306-2407

u.j.gerlach@bgk-hamburg.de

\section{Literatur}

[1] Carsenti-Etesse H, Doyon F, Desplaces $\mathrm{N}$ et al. Epidemiology of bacterial infection during management of open leg fractures. Eur J Clin Microbiol Infect Dis 1999; 18: 315-323

[2] Cierny GC, Mader JT, Pennick JJ. A clinical staging system for adult osteomyelitis. Contemp Orthop 1985; 10: 17-37

[3] Costerton JW, Stewart PS, Greenberg EP. Bacterial biofilms: a common cause of persistent infections. Science 1999; 248: $1318-1322$

[4] Gerlach U], Grimme C, Schoop R. Akute posttraumatische Osteitis. Trauma Berufskrankh 2009; 11 (Suppl. 2): 203-206

[5] Glombitza M. Der postoperative Frühinfekt. Trauma Berufskrankh 2014; 16 (Suppl. 4): 435-438

[6] Gristina AG. Biomaterial-centered infection: microbial adhesion versus tissue integration. Science 1987; 237: 1588-1595

[7] Gustilo RB, Anderson JT. Prevention of infection in the treatment of one thousand and twenty-five open fractures of long bones: retrospective and prospective analyses. J Bone Joint Surg Am 1976; 58: 453-458

[8] Stewart PS, Costerton JW. Antibiotic resistance of bacterias in biofilms. Lancet 2001; 358: 135-138

[9] Tiemann AH, Braunschweig R, Hofmann GO. Knocheninfektion. Unfallchirurg 2012; 115: 480-488

[10] Walter G, Kemmerer M, Kappler C et al. Behandlungsalgorithmen der chronischen Osteomyelitis. Dtsch Arztebl Int 2012; 109: $257-264$

Bibliografie

DOI https://doi.org/10.1055/s-0043-102266

OP-JOURNAL 2017; 33: 108-112 @ Georg Thieme Verlag KG Stuttgart · New York ISSN 0178-1715 